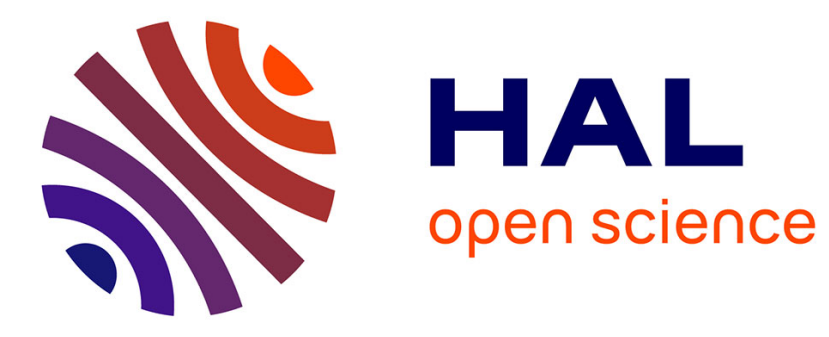

\title{
On the Reversibility of Live Equal-Conflict Petri Nets
}

\author{
Thomas Hujsa, Jean-Marc Delosme, Alix Munier Kordon
}

\section{To cite this version:}

Thomas Hujsa, Jean-Marc Delosme, Alix Munier Kordon. On the Reversibility of Live Equal-Conflict Petri Nets. 36th International Conference on Application and Theory of Petri Nets and Concurrency, PETRI NETS 2015, Jun 2015, Bruxelles, Belgium. pp.234-253, 10.1007/978-3-319-19488-2_12 . hal01197020

\section{HAL Id: hal-01197020 https: / hal.sorbonne-universite.fr/hal-01197020}

Submitted on 13 May 2019

HAL is a multi-disciplinary open access archive for the deposit and dissemination of scientific research documents, whether they are published or not. The documents may come from teaching and research institutions in France or abroad, or from public or private research centers.
L'archive ouverte pluridisciplinaire HAL, est destinée au dépôt et à la diffusion de documents scientifiques de niveau recherche, publiés ou non, émanant des établissements d'enseignement et de recherche français ou étrangers, des laboratoires publics ou privés. 


\title{
On the Reversibility of Live Equal-Conflict Petri Nets
}

\author{
Thomas Hujsa $^{1, \star}$, Jean-Marc Delosme ${ }^{2}$, and Alix Munier-Kordon ${ }^{3}$ \\ 1 LIAFA, Université Paris Diderot - Paris 7 \& CNRS UMR 7089, Paris, France \\ Thomas.Hujsa@liafa.univ-paris-diderot.fr \\ 2 Université d'Evry-Val-D'Essonne, IBISC, 91025, Evry, France \\ Jean-Marc.Delosme@ibisc.univ-evry.fr \\ 3 Sorbonne Universités, UPMC Paris 06, UMR 7606, LIP6, F-75005, Paris, France \\ Alix.Munier@lip6.fr
}

\begin{abstract}
A Petri net is reversible if its initial marking is a home marking, a marking reachable from any reachable marking. This property is fundamental in man-made systems as it lets a system return to its initial state using only internal operations.

Necessary and sufficient conditions are already known for the reversibility of well-formed Choice-Free and ordinary Free-Choice nets. Like the homogeneous Join-Free nets, these nets constitute subclasses of EqualConflict nets. In this larger class, the reversibility property is not well understood.

This paper provides the first characterization of reversibility for all the live Equal-Conflict systems by extending, in a weaker form, a known condition that applies to the Choice-Free and Free-Choice subclasses. We also show that this condition is tightly related to the Equal-Conflict class and does not apply to several other classes.
\end{abstract}

Keywords: Reversibility, home markings, liveness, weighted Petri nets, characterization, Equal-Conflict, Join-Free, Choice-Free, Free-Choice.

\section{Introduction}

Liveness and reversibility are behavioral properties of Petri nets that are fundamental for many real world applications. These systems (such as embedded or flexible manufacturing systems) have to keep all their functions (transitions) active over time, a condition modeled by the liveness property. These systems often also require a steady, regular, behavior and the possibility of returning to some particular states (markings) using only internal operations, a condition modeled by the reversibility property.

A system is live if any transition can be fired after a finite number of steps from any reachable marking. The markings that are reachable from every reachable marking-when they exist-are called home markings. A Petri net is reversible, or cyclic, if its initial marking is a home marking, in which case all

\footnotetext{
* The work of this author is supported by Digiteo / Project Tatami.
} 
reachable markings are home markings. Reversibility avoids a costly transient phase and favors a steady behavior from the start. Besides, it often simplifies substantially the study of the reachability graph.

Importance of Weights. In this study, we focus on weighted Petri nets, which are well suited to the modeling of real-life systems. In the domain of embedded systems, Synchronous Data Flow graphs [8], equivalent to particular weighted Petri nets, have been introduced to model the communications between a finite set of periodic processes. In the domain of flexible manufacturing systems (FMS), the weights make possible the modeling of bulk consumption or production of resources [15]. In these cases, weights allow a compact representation of the volumes of data or resources exchanged.

Important Weighted Subclasses. We focus on subclasses of weighted Petri nets that are defined by structural restrictions. A net is homogeneous if each place has all its outputs weights equal. The Equal-Conflict systems form a homogeneous subclass where transitions that have a common input place share the same set of input places.

This class generalizes several important subclasses of Petri nets. It contains the Choice-Free systems, also known as output non-branching systems [3], in which every place has at most one output transition. Weighted T-systemsequivalent to Synchronous Data Flow graphs - are Choice-Free systems where each place has at most one input transition. The homogeneous Join-Free Petri nets form a subclass of Equal-Conflict nets in which each transition has at most one input place. The homogeneous S-systems are homogeneous Join-Free systems in which each transition has at most one output place.

Previous Results. The problem of checking the reversibility property is decidable $[1,4]$, although its complexity is unknown. If the system is supposed to be bounded, a naive exponential algorithm would check the strong connectedness of its reachability graph. Moreover, neither one of the properties of liveness and reversibility implies the other [11].

The relation between liveness and reversibility has been studied in several weighted subclasses. The systems considered are often bounded, that is, with a bounded number of tokens in every place for all the reachable markings. Wellformedness is also commonly assumed for the net, ensuring the boundedness of the system for any initial marking and the existence of at least one live marking. Liveness and reversibility are equivalent for any well-formed T-system [14]. For well-formed Choice-Free systems, a characterization of reversibility was expressed in terms of the reversibility of particular subsystems under the liveness assumption in [7]. For the same class, a necessary and sufficient condition for the conjunction of liveness and reversibility was given in [15], which also applies to well-formed ordinary Free-Choice nets [5]. To our knowledge, no result of similar strength exists for homogeneous S-systems, hence for larger classes.

Nevertheless, for Equal-Conflict systems, some characterizations of liveness have been uncovered. Under the well-formedness assumption, there exist a struc- 
tural necessary and sufficient condition of liveness [17] and a checking method [16]. Also, the existence of reachable home markings is a necessary condition for the combined liveness and boundedness of an Equal-Conflict system [17].

Liveness and reversibility have been studied in many other classes, notably liveness in [2] and reversibility in [6].

Contributions. Our main contribution is a necessary and sufficient condition of reversibility for live, not necessarily bounded, Equal-Conflict systems. It is based on the existence of a feasible T-sequence, which is a sequence returning to the initial marking and where each transition is fired at least once.

The existence of a T-sequence is a necessary and sufficient condition for a well-formed Choice-Free system to be both live and reversible [15]. We exhibit a simple counter-example for the homogeneous S-system class. Consequently, the condition does not extend to the Equal-Conflict class.

We show easily that the existence of a feasible T-sequence is a necessary condition for a system to be both live and reversible. The major result is the proof that, for live Equal-Conflict systems, the existence of a T-sequence is also a sufficient condition of reversibility. We also provide various counter-examples showing that this characterization does not extend to several larger classes.

Organization of the Paper. In Section 2, we give general definitions, detail notations and properties of Petri nets, and define the subclasses that we study in the paper. In Section 3, we investigate the relationship between liveness and reversibility in weighted Petri nets and several bounded subclasses. We also introduce the notion of T-sequence and highlight its importance for the reversibility property. In Section 4, we explore a particular definition of fairness in EqualConflict nets and exploit it to prove the characterization of reversibility for all the live Equal-Conflict systems. In Section 5, we show by means of counterexamples that this characterization of reversibility does not extend to several classes of Petri nets. Finally, Section 6 is our conclusion.

\section{Definitions, Notations and Properties}

We first recall definitions and notations for weighted nets, markings, systems and firing sequences. Classical notions, such as liveness and boundedness, are formalized. Lastly, special classes of nets, including Choice-Free, Join-Free and Equal-Conflict nets, are recalled.

\subsection{Weighted and Ordinary Nets}

A (weighted) net is a triple $N=(P, T, W)$ where:

- the sets $P$ and $T$ are finite and disjoint, $T$ contains transitions and $P$ places,

- $W:(P \times T) \cup(T \times P) \mapsto \mathbb{N}$ is a weight function. 
$P \cup T$ is the set of the nodes of the net.

An arc leads from a place $p$ to a transition $t$ (respectively a transition $t$ to a place $p$ ) if $W(p, t)>0$ (respectively $W(t, p)>0$ ). An ordinary net is a net whose weight function $W$ has values in $\{0,1\}$.

The incidence matrix of a net $N=(P, T, W)$ is a place-transition matrix $C$ defined as

$$
\forall p \in P \quad \forall t \in T, \quad C[p, t]=W(t, p)-W(p, t)
$$

where the weight of each non-existing arc is 0 . The weight function $W$ can be represented by two place-transition matrices Pre and Post defined as follows: $\forall p \in P, \forall t \in T$, Pre $[p, t]=W(p, t)$ and Post $[p, t]=W(t, p)$. Consequently, the incidence matrix can be defined as $C=$ Post - Pre.

The pre-set of the element $x$ of $P \cup T$ is the set $\{w \mid W(w, x)>0\}$, denoted by ${ }^{\bullet} x$. By extension, for any subset $E$ of $P$ or $T,{ }^{\bullet} E=\bigcup_{x \in E} \cdot x$. The post-set of the element $x$ of $P \cup T$ is the set $\{y \mid W(x, y)>0\}$, denoted by $x^{\bullet}$. Similarly, $E^{\bullet}=\bigcup_{x \in E} x^{\bullet}$.

We denote by $\max _{p}^{N}$ the maximum output weight of $p$ in the net $N$. The simpler notation $\max _{p}$ is used when no confusion is possible.

A join-transition is a transition having at least two input places.

\subsection{Markings, Systems and Firing Sequences}

A marking $M$ of a net $N$ is a mapping $M: P \rightarrow \mathbb{N}$. A system is a couple $\left(N, M_{0}\right)$ where $N$ is a net and $M_{0}$ its initial marking.

A marking $M$ of a net $N$ enables a transition $t \in T$ if $\forall p \in{ }^{\bullet} t, M(p) \geq W(p, t)$. Generalizing to sets, a set $T$ of transitions is enabled by $M$ if every transition of $T$ is enabled by $M$. A marking $M$ enables a place $p \in P$ if $M(p) \geq \max _{p}$. Generalizing to sets, a set $P$ of places is enabled by $M$ if every place of $P$ is enabled by $M$.

The marking $M^{\prime}$ obtained from $M$ by firing an enabled transition $t$, denoted by $M \stackrel{t}{\rightarrow} M^{\prime}$, is defined by $\forall p \in P, M^{\prime}(p)=M(p)-W(p, t)+W(t, p)$.

A firing sequence $\sigma$ on the set of transitions $T$ is a mapping $\{1, \ldots, n\} \rightarrow T$ with $n \geq 1$, or $\mathbb{N} \rightarrow T$; it is finite of length $n$ in the first case and infinite otherwise. A firing sequence $\sigma=t_{1} t_{2} \cdots t_{n}$ is feasible if the successive markings obtained, $M_{0} \stackrel{t_{1}}{\longrightarrow} M_{1} \stackrel{t_{2}}{\longrightarrow} M_{2} \cdots \stackrel{t_{n}}{\longrightarrow} M_{n}$, are such that $M_{i-1}$ enables the transition $t_{i}$ for any $i \in\{1, \cdots, n\}$. We note $M_{0} \stackrel{\sigma}{\rightarrow} M_{n}$.

The Parikh vector $\vec{\sigma}: T \rightarrow \mathbb{N}$ associated with a finite sequence of transitions $\sigma$ maps every transition $t$ of $T$ to the number of occurrences of $t$ in $\sigma$.

A marking $M^{\prime}$ is said to be reachable from the marking $M$ if there exists a feasible firing sequence $\sigma$ such that $M \stackrel{\sigma}{\rightarrow} M^{\prime}$. The set of markings reachable from $M$ is denoted by $[M\rangle$. 
A home marking is a marking that can be reached from any reachable marking. Formally, $M$ is a home marking in the system $\left(N, M_{0}\right)$ if $\forall M^{\prime} \in$ $\left[M_{0}\right\rangle, M \in\left[M^{\prime}\right\rangle$. A system is reversible if its initial marking is a home marking.

\subsection{Liveness and Boundedness}

Liveness and boundedness are two basic properties ensuring that all transitions of a system $S=\left(N, M_{0}\right)$ can always be fired and that the overall number of tokens remains bounded. More formally,

- A system $S$ is live if for every marking $M$ in $\left[M_{0}\right\rangle$ and for every transition $t$, there exists a marking $M^{\prime}$ in $[M\rangle$ enabling $t$.

- $S$ is bounded if there exists an integer $k$ such that the number of tokens in each place never exceeds $k$. Formally, $\exists k \in \mathbb{N} \forall M \in\left[M_{0}\right\rangle \forall p \in P, M(p) \leq k$. $S$ is $k$-bounded if, for any place $p \in P, k \geq \max \left\{M(p) \mid M \in\left[M_{0}\right\rangle\right\}$.

- A system $S$ is well-behaved if it is live and bounded.

A marking $M$ is live (respectively bounded) for a net $N$ if the system $(N, M)$ is live (respectively bounded). The structure of a net $N$ may be studied to ensure the existence of an initial marking $M_{0}$ such that $\left(N, M_{0}\right)$ is live and bounded:

- $N$ is structurally live if a marking $M_{0}$ exists such that $\left(N, M_{0}\right)$ is live.

- $N$ is structurally bounded if the system $\left(N, M_{0}\right)$ is bounded for each $M_{0}$.

$-N$ is well-formed if it is structurally live and structurally bounded.

The algebraic properties of consistency and conservativeness are necessary conditions for well-formedness for all weighted Petri nets [10,13]. They are defined next in terms of the existence of particular annulers of the incidence matrix.

\subsection{Semiflows, Consistency and Conservativeness}

Semiflows are particular left or right annulers of an incidence matrix $C$ that is supposed to be non-empty:

- A P-semiflow is a non-null vector $X \in \mathbb{N}^{|P|}$ such that $X^{T} \cdot C=0$.

- A T-semiflow is a non-null vector $Y \in \mathbb{N}^{|T|}$ such that $C \cdot Y=0$.

We denote by $\mathcal{I}(V)$ the set of the indices of the vector $V$. The support of a vector $V$, denoted by $|V|$, is defined as the largest subset of $\mathcal{I}(V)$ being associated to non-zero components of $V$, meaning that $\forall i \in|V|, V[i] \neq 0$ and $\forall i \in \mathcal{I}(V) \backslash|V|$, $V[i]=0$. A P-semiflow is minimal if the greatest common divisor of its components is equal to 1 and its support is not a proper superset of the support of any other P-semiflow. Minimal T-semiflows are defined similarly.

We denote by $\mathbb{1}^{n}$ the column vector of size $n$ whose components are all equal to 1 . The conservativeness and consistency properties are defined as follows using the incidence matrix $C$ of a net $N$ :

- $N$ is conservative if a P-semiflow $X \in \mathbb{N}^{|P|}$ exists for $C$ such that $X \geq \mathbb{1}^{|P|}$.

- $N$ is consistent if a T-semiflow $Y \in \mathbb{N}^{|T|}$ exists for $C$ such that $Y \geq \mathbb{1}^{|T|}$.

The net on Figure 1 is conservative and consistent. 

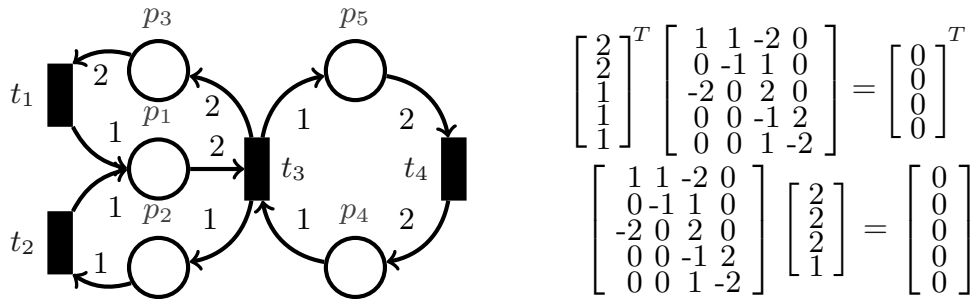

Fig. 1. This weighted net is conservative (the left vector $[2,2,1,1,1]$ is a $\mathrm{P}$-semiflow and its components are $\geq 1$ ) and consistent (the right vector $[2,2,2,1]^{T}$ is a T-semiflow and its components are $\geq 1$ ).

\subsection{Choice-Free Nets, Join-Free Nets and Subclasses}

The following basic subclasses of weighted Petri nets are defined by structural restrictions on the number of inputs or outputs of nodes. By studying these particular structures, the understanding of the behavior has been improved in several larger classes $[12,17]$.

In Choice-Free nets, each place has at most one output transition, meaning that choices are not allowed. More formally, $N=(P, T, W)$ is a Choice-Free net if $\forall p \in P,\left|p^{\bullet}\right| \leq 1$.

In Join-Free nets, each transition has at most one input place, meaning that synchronizations are not allowed. More formally, $N=(P, T, W)$ is a Join-Free net if $\forall t \in T,|\bullet t| \leq 1$.

The net of Figure 1 is Choice-Free but not Join-Free: $t_{3}$ is a join-transition.

A net $N$ is a Fork-Attribution net (or FA net) if it is a Choice-Free net and a Join-Free net. A net is an $S$-net if every transition has at most one input and one output. A net is a T-net if every place has at most one input and one output.

\subsection{Equal-Conflict Relation, Sets, Nets and Larger Classes}

In order to consider nets that are more expressive than the basic Choice-Free or Join-Free classes, some choices or synchronizations must be allowed.

However, in presence of structural choices, the behavior depends on the resolution of conflicts, which is limited by the preconditions of the conflicting transitions and by the current marking. When these preconditions are identical, all the alternatives are equivalent and the study of the behavior is simplified.

This notion of equal preconditions is captured by the next relation on the transitions of any weighted net, which was defined in [17].

Let $N=(P, T, W)$ be a net. Two transitions $t, t^{\prime}$ of $T$ are in equal conflict relation if $\operatorname{Pre}[P, t]=\operatorname{Pre}\left[P, t^{\prime}\right] \neq \mathbb{O}^{|P|}$, where $\operatorname{Pre}[P, t]$ denotes the $t$-th column of the matrix Pre. It is an equivalence relation on the set of transitions, and each equivalence class is an equal conflict set. 
We deduce that an equal conflict set is enabled by a marking $M$ if and only if at least one transition of this set is enabled by $M$.

A net $N=(P, T, W)$ is an Equal-Conflict (EC) net if for all transitions $t$ and $t^{\prime}$ of $N, \bullet t \cap \bullet t^{\prime} \neq \varnothing \Rightarrow \operatorname{Pre}[P, t]=\operatorname{Pre}\left[P, t^{\prime}\right]$.

A consequence of this definition is that Equal-Conflict nets are homogeneous, meaning that for every place $p$, all the output weights of $p$ are equal. Figure 2 contains an Equal-Conflict net on the left.

The Equal-Conflict class strictly extends the expressiveness of Choice-Free nets by adding the possibility to model choices that are equally favored.
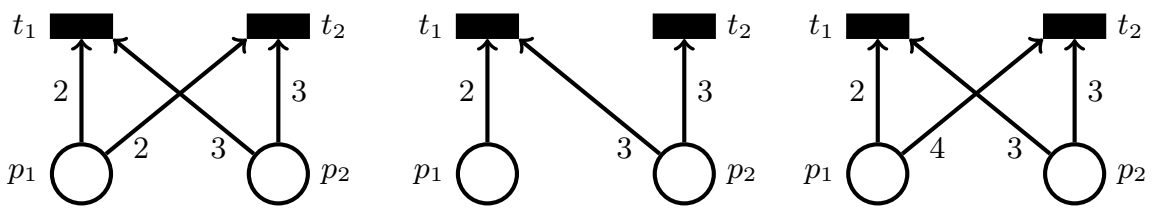

Fig. 2. The net on the left is an Equal-Conflict net. In the middle, ${ }^{\bullet} t_{1}=\left\{p_{1}, p_{2}\right\} \neq$ $\left\{p_{2}\right\}={ }^{\bullet} t_{2}$, hence the net is not Equal-Conflict. On the right, the pre-sets of both transitions are equal, however it is not Equal-Conflict since it is not homogeneous: the output weights of $p_{1}$ are not all equal.

Finally, we recall the following well-known classes, whose weighted versions generalize the Equal-Conflict class.

Free-Choice nets are ordinary (unit-weighted) Equal-Conflict nets. The weighted generalization of this class encompasses the Equal-Conflict nets and is depicted on the right in Figure 2.

A net $N=(P, T, W)$, either ordinary or weighted, is Asymmetric-Choice if $\forall p_{1}, p_{2} \in P, p_{1}^{\bullet} \cap p_{2}^{\bullet} \neq \varnothing \Rightarrow p_{1}^{\bullet} \subseteq p_{2}^{\bullet}$ or $p_{2}^{\bullet} \subseteq p_{1}^{\bullet}$. A weighted homogeneous Asymmetric-Choice net is shown in the middle of Figure 2.

Figure 3 represents the inclusion relations between the special subclasses of weighted Petri nets considered in this paper.

\section{Liveness, Reversibility and T-sequences}

We recall known results and provide examples that explain some interactions between liveness and reversibility in weighted subclasses. We then introduce the notion of T-sequence and study its importance in relation with these properties.

\subsection{Previous Results on the Reversibility of Live Systems}

Since we are interested in systems that are both live and reversible, we first illustrate some relations between these properties. While, under the well-formedness 


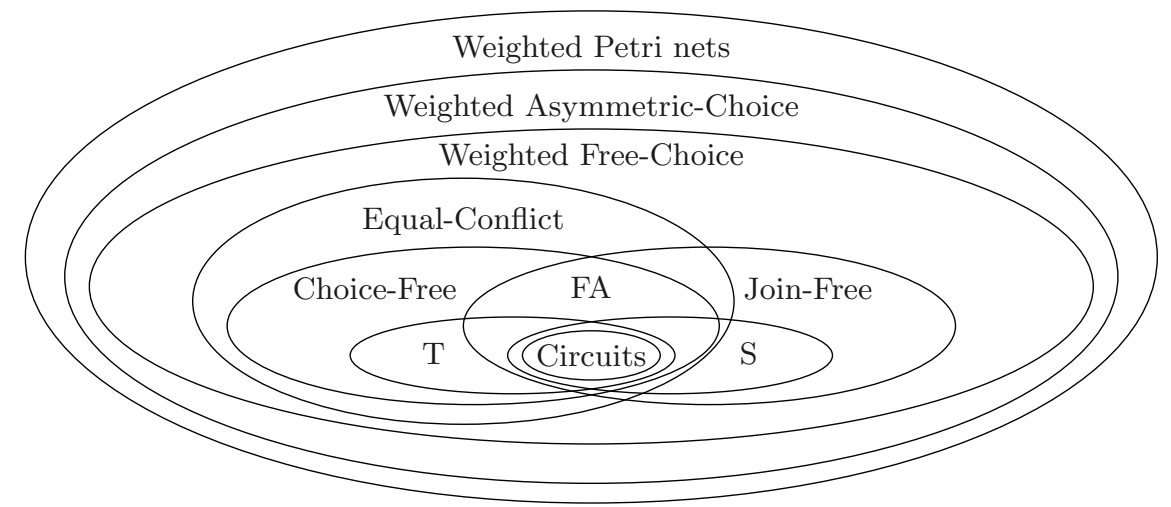

Fig. 3. Some classes and subclasses of weighted systems.

assumption, liveness is equivalent to reversibility in weighted T-systems [14], it does not imply reversibility in weighted Fork-Attribution systems and homogeneous S-systems, as illustrated in Figure 4.
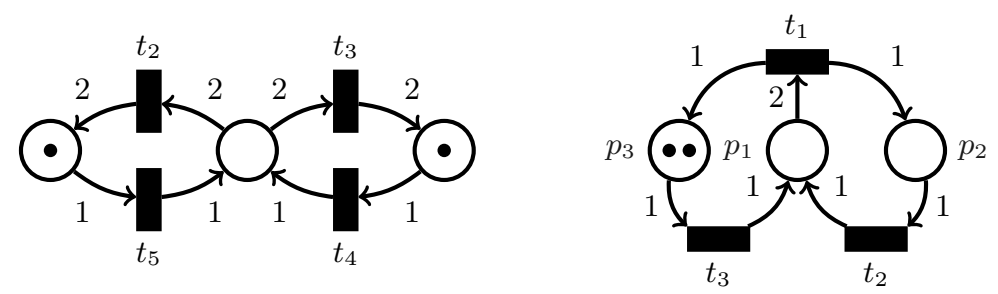

Fig. 4. On the left, a live S-system. On the right, a live Fork-Attribution system. None of them is reversible.

Thus, since a live system may not be reversible, other notions, such as Tsequences, must be introduced to study the reversibility property.

\section{$3.2 \quad$ T-sequences}

We introduce next the notion of T-sequence and show that the existence of such a sequence is necessary to have both liveness and reversibility.

Definition 1 (T-sequences, partial T-sequences). Consider a Petri net with set of transitions T. A T-sequence is a sequence whose Parikh vector is equal to a T-semiflow whose support is T. A partial T-sequence is a sequence whose Parikh vector is equal to a T-semiflow whose support is different from $T$.

The alternative expressions feasible or realizable T-semiflow may be found in the literature when there exists a feasible (partial or not) T-sequence. Such 
a sequence, when feasible at the initial marking, defines weak reversibility in [14].

The next lemma provides a necessary condition to obtain both liveness and reversibility.

Lemma 1. If a system $S=\left(N, M_{0}\right)$ is live and reversible, then it enables a T-sequence.

Proof. Suppose that the system is live and reversible. By the liveness assumption, there exists a feasible sequence $\sigma_{0}$ whose support is the set of all transitions. By the reversibility assumption, there exists a feasible sequence $\sigma_{1}$ returning to $M_{0}$. Thus, the feasible sequence $\sigma_{0} \sigma_{1}$ is a feasible T-sequence.

Consequently, any live and reversible Petri net is consistent.

In the other direction, the existence of a feasible T-sequence implies both liveness and reversibility in (weighted) Choice-Free nets [15] and (ordinary) FreeChoice nets [5] under the well-formedness assumption. However this implication is false in general. Indeed, a well-formed homogeneous S-system may have a feasible T-sequence while it is neither live nor reversible, as illustrated in Figure 5.

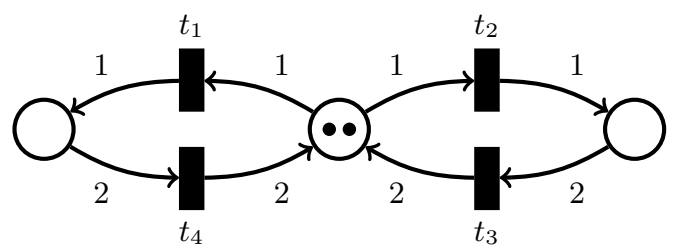

Fig. 5. This well-formed homogeneous S-system is not live (fire $t_{1} t_{2}$ ) although a Tsequence is feasible (fire $t_{1} t_{1} t_{4} t_{2} t_{2} t_{3}$ ).

Other particular classes have been studied in [9], which investigates the relationship between the reversibility property and the existence of reachable markings that enable a partial T-sequence associated to a minimal T-semiflow.

This fact justifies the study of reversibility under liveness hypothesis in the next section. We also show in that section that the existence of a feasible Tsequence is sufficient for reversibility in the Equal-Conflict class when liveness is assumed.

\section{Reversibility of Live Equal-Conflict Systems}

Under the liveness hypothesis, we investigate the reversibility property in EqualConflict systems, which may be unbounded. First, we define a notion of fairness 
and develop an associated property for sequences. Then, we use the fairness to facilitate the proof of the characterization of reversibility for all live EqualConflict systems.

\subsection{Fairness in Equal-Conflict Systems}

Taking inspiration from [17], we define a fairness property adapted to the EqualConflict class. Then, we present a result about fairness that will prove useful for the study of the reversibility property.

Definition 2 (Fairness in Equal-Conflict systems). An infinite firing sequence is globally fair if it fires every transition of the system an infinite number of times. An infinite firing sequence is locally fair if

- when an equal conflict set contains a transition that is fired an infinite number of times, all of its transitions are fired an infinite number of times, and

- when an equal conflict set is enabled, one of its transitions is fired after a finite number of firings.

The following theorem is similar to a result of [17] which uses a slightly different definition of fairness. Comparing with [17], we replace the boundedness and strong connectedness assumptions by the liveness assumption.

Theorem 1 (Fairness in live Equal-Conflict systems). Let $S$ be a live Equal-Conflict system. An infinite sequence $\sigma$ that is feasible in $S$ is globally fair if and only if it is locally fair.

Proof. If $\sigma$ is globally fair, it is easy to see that $\sigma$ is locally fair. Let us prove the converse. Suppose that $\sigma$ is locally fair.

Denote by $Q$ the set of the equal conflict sets containing a transition that occurs infinitely often in $\sigma$ and by $\bar{Q}$ the set of the other equal conflict sets. The set $Q$ is non-empty since there is only a finite number of equal conflict sets and $\sigma$ is infinite. If $\bar{Q}$ is empty, then we are done. Now suppose that $\bar{Q}$ is non-empty.

By definition of $Q$ and by the local fairness assumption, all the transitions of the sets in $Q$ are fired an infinite number of times in $\sigma$, while all the transitions of the sets in $\bar{Q}$ are fired a finite number of times and become forever non-enabled after the firing of a finite prefix sequence $\sigma_{0}$ of $\sigma$. Denote by $M$ the marking reached by firing $\sigma_{0}$ in $S$ and by $\sigma^{\prime}$ the infinite suffix sequence of $\sigma$ satisfying $\sigma=\sigma_{0} \sigma^{\prime}$.

By the liveness assumption, there exists a transition $t$ in $\bar{Q}$ and a finite sequence $\sigma_{1}$ feasible at $M$ such that $\sigma_{1}$ contains only transitions of $Q$ and enables $t$. The sequence $\sigma_{1}$ may not be a prefix of $\sigma^{\prime}$, however all the transitions of $Q$ are fired an infinite number of times in $\sigma^{\prime}$. We deduce that a finite prefix sequence $\sigma_{2}$ of $\sigma^{\prime}$ exists such that $\vec{\sigma}_{2} \geq \vec{\sigma}_{1}$. Moreover, since only transitions of $\bar{Q}$ are structurally allowed to remove tokens from the inputs of $t$, the transition $t$ becomes enabled after the firing of the finite sequence $\sigma_{0} \sigma_{2}$, contradicting the fact that every transition of $\bar{Q}$ stays forever non-enabled after the firing of $\sigma_{0}$. Thus, $\bar{Q}$ is empty and $\sigma$ is globally fair. 
In the following, we will use fair sequences to study the reversibility of live Equal-Conflict systems.

\subsection{A Characterization of Reversibility under the Liveness Assumption}

By Lemma 1, in every live Petri net, the existence of a feasible T-sequence is necessary for reversibility. We show that it is also sufficient for the class of live Equal-Conflict nets.

To obtain the sufficiency, we show that after the firing of any feasible sequence, we can use the T-sequence to construct another sequence that leads to the initial marking.

Starting from an initial marking, any firing sequence that is a prefix of a feasible T-sequence can be trivially completed to reach the initial marking again. More generally, any firing sequence that solves conflicts by following the local ordering induced by the adequate multiple of the T-sequence can be completed to reach the initial marking. However, if a transition is fired that solves a conflict by following a different ordering, the possibility to reach the initial marking is not ensured anymore. The occurrences of other transitions in the same conflicting set that should have been fired earlier are called delayed occurrences.

The proof of the characterization is constructive and makes use of two algorithms that compute this sequence. The first algorithm (Algorithm 1) fires at least all the delayed occurrences and returns the corresponding sequence $\sigma_{t}$. The second algorithm (Algorithm 2) starts after the end of the first algorithm and builds a sequence $\sigma_{t}^{\prime}$ returning to the initial marking. These two sequences are illustrated in Figure 6.

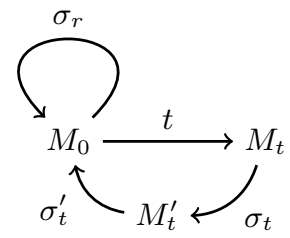

Fig. 6. If the T-sequence $\sigma_{r}$ is feasible and $t$ is fired, then Algorithm 1 builds the sequence $\sigma_{t}$ and Algorithm 2 computes the sequence $\sigma_{t}^{\prime}$, which returns to the initial marking.

Notations. For every transition $t$, we denote by $E^{t}$ the equal conflict set containing $t$. We introduce $\sigma^{n}, n$ being a positive integer, to denote the concatenation of the sequence $\sigma$ taken $n$ times, and represent its infinite concatenation by $\sigma^{\infty}$.

The notation $K_{t_{i}}^{n}(\sigma), n \geq 1$, or more simply $K_{i}^{n}(\sigma)$, denotes the largest prefix sequence of $\sigma$ preceding the $n$-th occurrence of $t_{i}$ in $\sigma$, thus containing $n-1$ 
occurrences of $t_{i}$. For example, considering the sequence $\sigma=t_{1} t_{2} t_{1} t_{3} t_{1} t_{2} t_{3}$, $K_{t_{1}}^{3}(\sigma)=t_{1} t_{2} t_{1} t_{3}$ and $K_{t_{3}}^{1}(\sigma)=t_{1} t_{2} t_{1}$.

Consider an equal-conflict set $E$ and sequences $\tau$ and $\kappa$ such that $\vec{\tau}<\vec{\kappa}$. Assume there exists a transition $t$ in $E$ for which $\vec{\tau}(t)<\vec{\kappa}(t)$. Consider for each transition $t^{\prime}$ in $E$ such that $\vec{\tau}\left(t^{\prime}\right)<\vec{\kappa}\left(t^{\prime}\right)$, its next occurrence in $\kappa$ after its $\vec{\tau}\left(t^{\prime}\right)$-th occurrence. The transition $t^{\prime}$ in $E$ whose next occurrence is the first to appear in $\kappa$ is returned by a function, called the next transition function and denoted by $\operatorname{tnext}(E, \tau, \kappa)$. Figure 7 illustrates these notations.

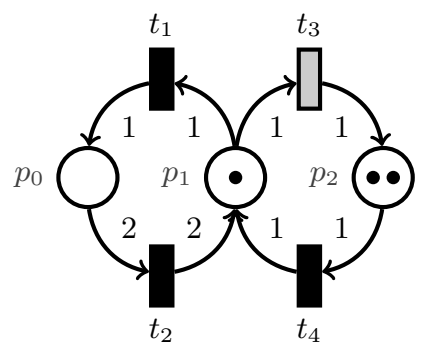

Fig. 7. The equal conflict sets are $E^{t_{1}}=\left\{t_{1}, t_{3}\right\}, E^{t_{2}}=\left\{t_{2}\right\}$ and $E^{t_{4}}=\left\{t_{4}\right\}$. Consider the feasible sequence $\sigma=t_{4} t_{4} t_{1} t_{3} t_{1} t_{2} t_{3}$. The subsequences of $\sigma$ obtained by projection on each set, $\sigma_{1}=t_{1} t_{3} t_{1} t_{3}, \sigma_{2}=t_{2}$ and $\sigma_{4}=t_{4} t_{4}$, define local orderings. Define $\tau=t_{3} t_{4} t_{1}$. Then $\vec{\sigma}>\vec{\tau}$, and the next transition to be fired in $E^{t_{1}}$ is the one whose next occurrence appears first in $\sigma_{1}$. Since $\vec{\tau}\left(t_{1}\right)=1$ and $\vec{\tau}\left(t_{3}\right)=1$, we deduce that $\operatorname{tnext}\left(E^{t_{1}}, \tau, \sigma\right)=t_{1}$.

Algorithm 1 determines a way of firing the delayed occurrences while following the local ordering induced by the T-sequence in every other equal conflict set. Lemma 2 shows the termination of this algorithm. Then, Lemma 3 provides an equality indicating a match between occurrence counts.

The next technical lemma proves the termination of Algorithm 1, which computes a particular sequence $\sigma_{t}$ and is illustrated in Figure 8.

Lemma 2. Let $\left(N, M_{0}\right)$ be a live Equal-Conflict system in which a T-sequence $\sigma_{r}$ is feasible. Then, for every transition $t$ enabled by $M_{0}$, with $M_{0} \stackrel{t}{\longrightarrow} M_{t}$, Algorithm 1 terminates and computes the sequence $\sigma_{t}$ that is feasible at $M_{t}$.

Proof. Consider the marking $M_{t}$ reached by firing a transition $t$ from $M_{0}$. We prove that Algorithm 1 computes such a sequence $\sigma_{t}$ that is feasible at $M_{t}$.

The objective of the outer loop is to fire the transitions different from $t$ in $E^{t}$ until the number of their occurrences in $\tau$ equals that in $\kappa_{0}$. Every time $E^{t}$ is enabled, a firing occurs in this set that follows the order of $\kappa_{0}$ until completion.

The objective of the inner loop is to fire transitions that do not belong to $E^{t}$ by following the associated order in $\left(\sigma_{r}\right)^{\infty}$ so as to enable $E^{t}$. 


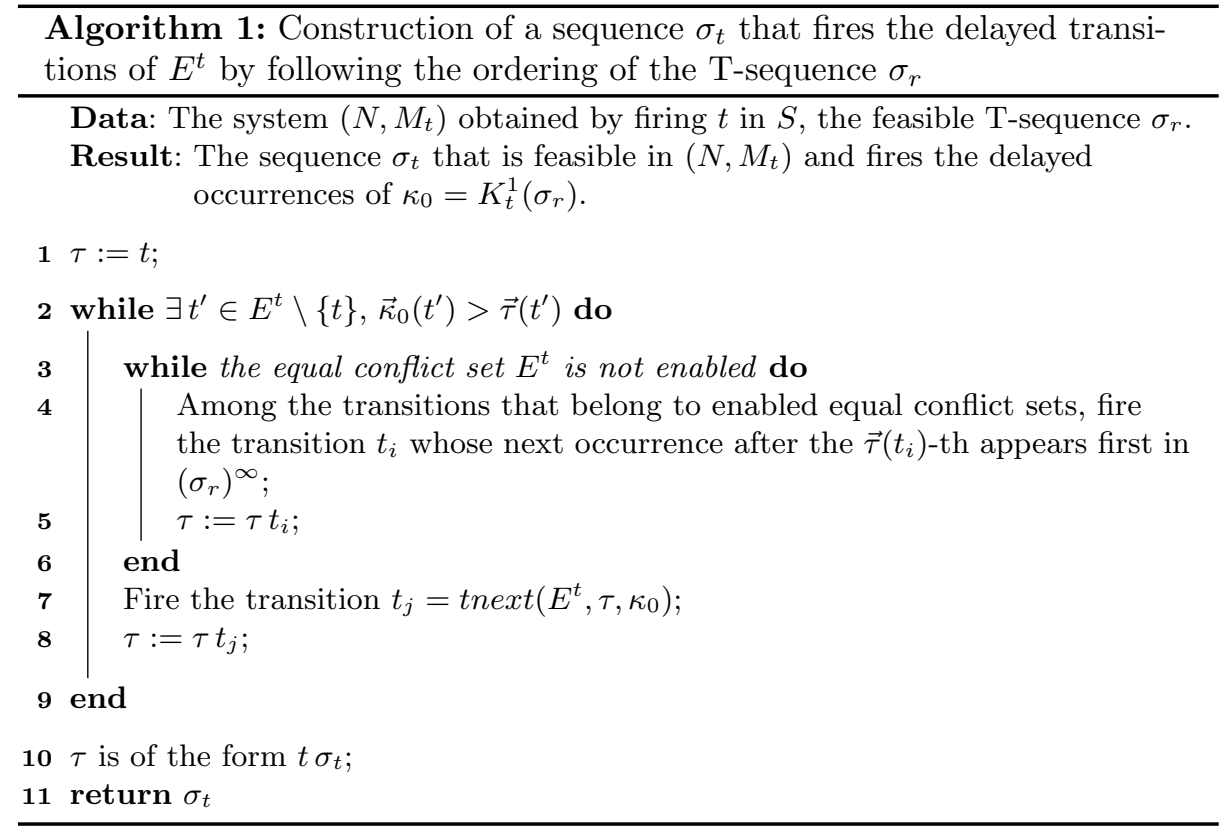

Let us show that the inner loop always terminates and enables $E^{t}$. First, by the liveness assumption, every reachable marking enables at least one equal conflict set. Now suppose that the inner loop does not terminate. Consequently, an infinite feasible sequence $\tau$ is fired that never enables $E^{t}$. Since the firings in the loop follow the order of $\left(\sigma_{r}\right)^{\infty}$ and the support of $\sigma_{r}$ is $T$, the sequence $\tau$ is locally fair, thus globally fair by Theorem 1 , contradicting the fact that $E^{t}$ never becomes enabled. We deduce that $E^{t}$ becomes enabled and the inner loop terminates.

We now prove the termination of the algorithm. Since the inner loop always terminates, a transition $t_{j}$ is fired at the end of every iteration of the outer loop such that $\vec{\kappa}_{0}\left(t_{j}\right)>\vec{\tau}\left(t_{j}\right)$ and $t_{j}$ is concatenated to the current $\tau$, decreasing the number of remaining steps to attain $\vec{\kappa}_{0}\left(t_{j}\right)$. Hence the outer loop terminates.

In Algorithm 1, the firings that did not belong to $E^{t}$ followed the order of $\sigma_{r}^{\infty}$. At the end, there is no delayed occurrence of any transition in $E^{t}$. We deduce the next property on the number of occurrences in $\tau$.

Lemma 3 (Property of $\left.\tau=t \sigma_{t}\right)$. Let $S=\left(N, M_{0}\right)$ be a live Equal-Conflict system in which a T-sequence $\sigma_{r}$ is feasible. Consider the sequence $\sigma_{t}$ constructed by Algorithm 1 after the firing of any transition $t$ in $S$. Consider the sequences $\tau=t \sigma_{t}$ and $\kappa=\sigma_{r}^{\alpha}$ where $\alpha \geq 1$ is the smallest integer such that $\vec{\tau} \leq \alpha \cdot \vec{\sigma}_{r}$. Then, for each equal-conflict set $E$ such that $t_{u}=\operatorname{tnext}(E, \tau, \kappa)$ is defined, with $m=\vec{\tau}\left(t_{u}\right)+1$ and $K_{u}=K_{u}^{m}(\kappa)$, and for every transition $t^{\prime} \in E, \vec{\tau}\left(t^{\prime}\right)=\vec{K}_{u}\left(t^{\prime}\right)$. For every other equal-conflict set $E$, for each transition $t^{\prime}$ in $E, \vec{\tau}\left(t^{\prime}\right)=\vec{\kappa}\left(t^{\prime}\right)$. 

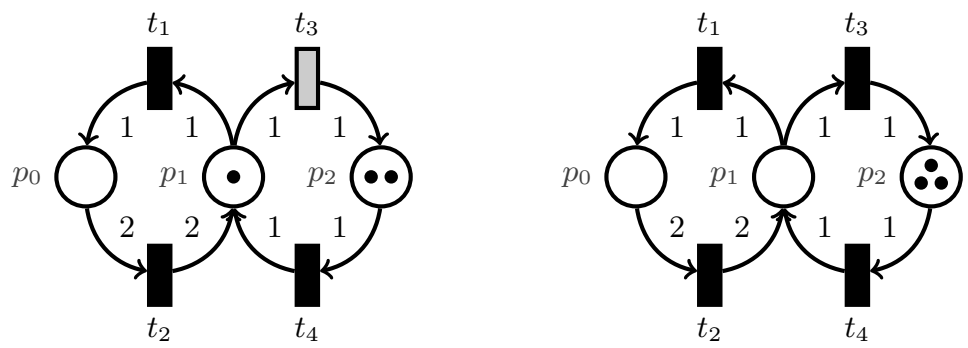

Fig. 8. Consider the T-sequence $\sigma_{r}=t_{1} t_{4} t_{1} t_{2} t_{3}$, which is feasible for the system $\left(N, M_{0}\right)$ on the left. Setting $t=t_{3},\left(N, M_{t}\right)$ is pictured on the right. Since the first output transition of $p_{1}$ to be fired in $\sigma_{r}$ is $t_{1} \neq t_{3}$, two occurrences of $t_{1}$ are delayed. Starting from the system on the right, Algorithm 1 constructs the sequence $\sigma_{t}$ that fires the delayed occurrences while following the local ordering in every other place. Before the loop, $\tau=t_{3}$ and $\kappa_{0}=K_{t_{3}}^{1}\left(\sigma_{r}\right)=t_{1} t_{4} t_{1} t_{2}$. The sequence computed is $\sigma_{t}=t_{4} t_{1} t_{4} t_{1}$.

Proof. Algorithm 1 terminates by Lemma 2. At the end of the outer loop, for every equal-conflict set $E$ such that $t_{u}=\operatorname{tnext}(E, \tau, \kappa)$ with $\vec{\tau}\left(t_{u}\right)<\vec{\kappa}\left(t_{u}\right)$, two cases have to be considered.

If $t_{u}$ does not belong to $E^{t}$, then all firings of $E$ appeared in the same order and are as many in $\tau$ as in $K_{u}$ in the inner loop. We deduce that every transition $t^{\prime}$ of $E$ satisfies $\vec{\tau}\left(t^{\prime}\right)=\vec{K}_{u}\left(t^{\prime}\right)$. Otherwise, $t_{u}$ belongs to $E^{t}$ and the first loop fired precisely all the occurrences of $E^{t}$ that belong to $\kappa_{0}$, in addition to the first unique firing of $t$. Thus, every transition $t^{\prime}$ of $E^{t}$ satisfies $\vec{\tau}\left(t^{\prime}\right)=\vec{K}_{u}\left(t^{\prime}\right)$.

Finally, in every other equal-conflict set, there is no transition $t_{u}$ such that $\vec{\tau}\left(t_{u}\right)<\vec{\kappa}\left(t_{u}\right)$. Since $\vec{\tau} \leq \vec{\kappa}$, we deduce the second equality.

At the end of Algorithm 1, take the example of Figure 8, with $E^{t_{1}}=\left\{t_{1}, t_{3}\right\}$, $E^{t_{2}}=\left\{t_{2}\right\}, E^{t_{4}}=\left\{t_{4}\right\}, \tau=t_{3} \sigma_{t}=t_{3} t_{4} t_{1} t_{4} t_{1}$ and $\kappa=\left(\sigma_{r}\right)^{2}$.

For $E^{t_{1}}, \operatorname{tnext}\left(E^{t_{1}}, \tau, \kappa\right)=t_{1}, K_{1}=t_{1} t_{4} t_{1} t_{2} t_{3}, \vec{\tau}\left(t_{1}\right)=2=\vec{K}_{1}\left(t_{1}\right)$ and $\vec{\tau}\left(t_{3}\right)=1=\vec{K}_{1}\left(t_{3}\right)$.

For $E^{t_{2}}, \operatorname{tnext}\left(E^{t_{2}}, \tau, \kappa\right)=t_{2}, K_{2}=t_{1} t_{4} t_{1}$ and $\vec{\tau}\left(t_{2}\right)=0=\vec{K}_{2}\left(t_{2}\right)$.

For $E^{t_{4}}$, the second equality of the lemma is satisfied: $\vec{\tau}\left(t_{4}\right)=2=\vec{\kappa}\left(t_{4}\right)$.

Using Lemma 3, the next theorem shows that Algorithm 2 builds a sequence $\sigma_{t}^{\prime}$ that is feasible after the firing of $\tau=t \sigma_{t}$ and reaches the initial marking. The sequence is illustrated in Figure 6 and an application of this second algorithm is presented in Figure 9.

Theorem 2. Let $S=\left(N, M_{0}\right)$ be a live Equal-Conflict system, with $N=$ $(P, T, W)$. Suppose there exists a feasible T-sequence $\sigma_{r}$ in $S$. For every transition $t$ enabled by $M_{0}$ such that $M_{0} \stackrel{t}{\longrightarrow} M_{t}$, there exists a sequence $\sigma^{\star}$ that is feasible at $M_{t}$ such that $\sigma=t \sigma^{\star}$ is a T-sequence satisfying $\vec{\sigma}=k \cdot \vec{\sigma}_{r}$ for some integer $k \geq 1$. 


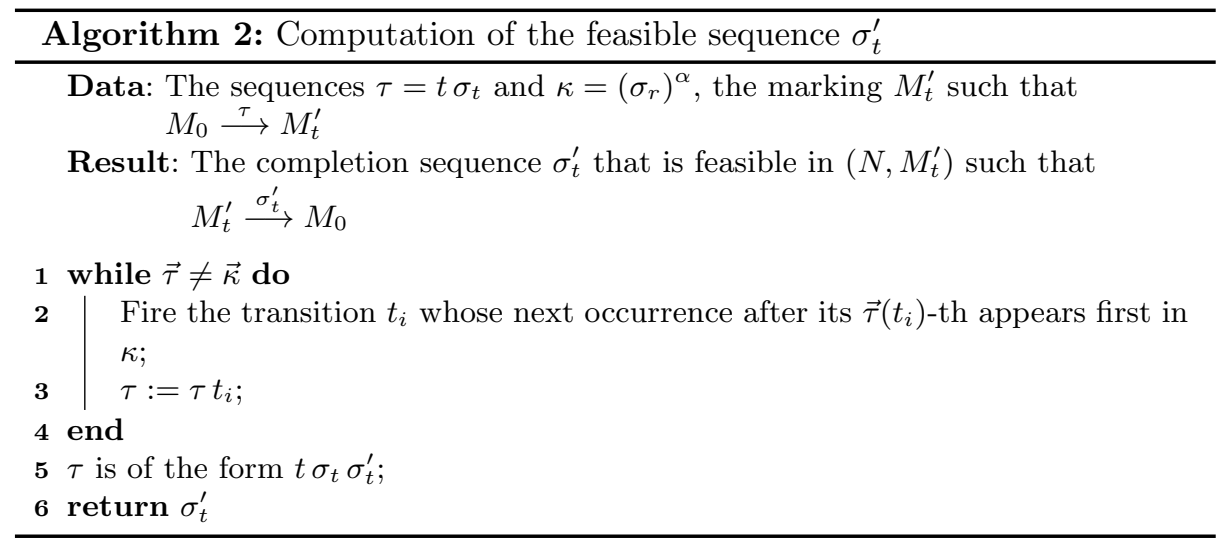

Proof. In the rest of the proof, we note $\kappa_{0}=K_{t}^{1}\left(\sigma_{r}\right)$ the largest prefix sequence of $\sigma_{r}$ preceding the first occurrence of $t$, meaning that $\sigma_{r}$ is of the form $\kappa_{0} t \sigma_{2}$, while the sequence $\kappa_{0}$ does not contain any occurrence of $t$. This sequence is well-defined since the support of $\vec{\sigma}_{r}$ is $T$.

If $t$ is the first transition of $E^{t}$ to be fired following the order of $\sigma_{r}$, meaning that $\kappa_{0}$ does not contain any occurrence of transitions in $E^{t}$, then the sequence $\kappa_{0}$ does not use any token from the input places of $t$, thus one can execute $\kappa_{0}$ after the firing of the first occurrence of $t$ and the sequence $t \kappa_{0} \sigma_{2}$ is feasible at $M_{0}$. Hence, $\sigma^{\star}=\kappa_{0} \sigma_{2}$.

Otherwise, $t$ is not the first transition in $E^{t}$ to be fired following the order of $\sigma_{r}$, meaning that $\kappa_{0}$ contains at least one occurrence of another transition of $E^{t}$. We show next that Algorithm 2, whose inputs are the sequences computed by Algorithm 1, completes $\tau$ up to $\kappa$ by following the order of the remaining unfired occurrences in $\kappa$. We deduce that the sequence $\sigma^{\star}$ obtained at the end reaches the initial marking.

To achieve this objective, we prove that the following loop invariant $I(k)$ is true for $k \geq 0$ :

$I(k)$ : "at the end of iteration $k$, for every transition $t_{u}$ such that $\vec{\tau}\left(t_{u}\right)<\vec{\kappa}\left(t_{u}\right)$ and $t_{u}=\operatorname{tnext}\left(E^{t_{u}}, \tau, \kappa\right)$, then for every transition $t_{j}$ of $E^{t_{u}}, \vec{\tau}\left(t_{j}\right)=\vec{K}\left(t_{j}\right)$, where $K$ denotes the sequence $K_{u}^{m}(\kappa)$ and $m$ is the value $\vec{\tau}\left(t_{u}\right)+1$ ".

Before starting the loop, $k=0$ and Lemma 3 applies.

Now assume that $k$ iterations of the loop occurred and $I(k)$ is true. During iteration $k+1$, a new transition $t_{i}$ is fired following the order of $\kappa$. At the end of iteration $k+1$, for every transition $t_{u}$ such that $\vec{\tau}\left(t_{u}\right)<\vec{\kappa}\left(t_{u}\right)$ and $t_{u}=$ $\operatorname{tnext}\left(E^{t_{u}}, \tau, \kappa\right)$, we denote by $K^{\prime}$ the sequence $K_{u}^{m^{\prime}}(\kappa)$ where $m^{\prime}=\vec{\tau}\left(t_{u}\right)+1$ and consider two cases. First, if $t_{u}$ does not belong to $E^{t_{i}}$, then $K^{\prime}$ is the same sequence as in the previous iteration and for every transition $t_{j}$ of $E^{t_{u}}, \vec{\tau}\left(t_{j}\right)$ has not changed either, thus $\vec{\tau}\left(t_{j}\right)=\vec{K}^{\prime}\left(t_{j}\right)$. Otherwise, if $t_{u}$ belongs to $E^{t_{i}}$, implying $E^{t_{i}}=E^{t_{u}}$, then $K^{\prime}$ contains the same number of occurrences of every transition $t_{j}$ of $E^{t_{i}}$ as in the sequence $K$ associated to $t_{i}$ in the previous iteration, 
except for $t_{i}$, whose number has been incremented by one. Besides, the only transition whose number of occurrences in $\tau$ has been incremented by one is $t_{i}$. Consequently, for every transition $t_{j}$ of $E^{t_{u}}$, we have $\vec{\tau}\left(t_{j}\right)=\vec{K}^{\prime}\left(t_{j}\right)$. We deduce finally that all the equalities that are supposed to be true at the end of iteration $k$ remain true at the end of iteration $k+1$.

Hence, the invariant is true at every iteration of the loop. Furthermore, by definition of the $t_{i}$ chosen at every step, for which we define the current value $m=\vec{\tau}\left(t_{i}\right)+1$ and the sequence $K=K_{i}^{m}(\kappa)$, all the occurrences in $K$ are already present in the sequence $\tau$ of the current iteration. Thus, at the beginning of every iteration, for every transition $t_{j} \in T, \vec{\tau}\left(t_{j}\right) \geq \vec{K}\left(t_{j}\right)$.

Moreover, the sequence $K$ is feasible at $M_{0}$ and leads to a marking that enables $t_{i}$, by definition of the feasible sequence $\kappa$. Thus, $\tau$ fired the input transitions of the input places of $t_{i}$ at least as many times as in $K$. Then, the invariant implies that the transitions of $E^{t_{i}}$ fired exactly as many times in $K$ as in $\tau$. Thus, the input places of $t_{i}$ received at least the number of tokens they would receive by firing $K$ from $M_{0}$, implying that $t_{i}$ is enabled.

We deduce that the loop completes $\vec{\tau}$ up to $\vec{\kappa}$ and terminates.

Finally, since $\kappa$ is of the form $\left(\sigma_{r}\right)^{\alpha}$ for some integer $\alpha>0$, the feasible sequence $t \sigma^{\star}$ is a $\mathrm{T}$-sequence.

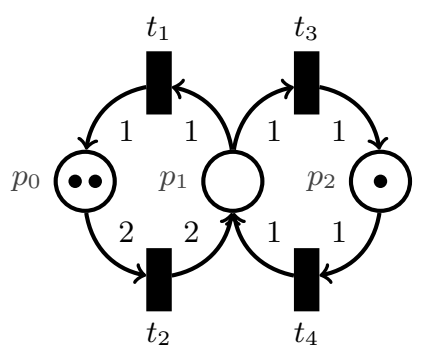

$$
\begin{aligned}
& \kappa=\left(\sigma_{r}\right)^{2}=t_{1} \quad t_{4} \quad t_{1} \quad t_{2} \quad t_{3} \begin{array}{llllllllllll}
t_{1} & t_{1} & t_{2} & t_{3}
\end{array} \\
& \times \times \times \times \times \\
& \tau=t_{3} \sigma_{t}=t_{3} \quad t_{4} \quad t_{1} \quad t_{4} \quad t_{1} \\
& \sigma_{t}^{\prime}=t_{2} \quad t_{1} \quad t_{1} \quad t_{2} \quad t_{3}
\end{aligned}
$$

Fig. 9. On the left, the system obtained at the end of Algorithm 1 and the corresponding value of $\tau$ on the right. The crosses indicate the occurrences of transitions in $\kappa$ that have been fired in $\tau=t \sigma_{t}$, setting $t=t_{3}$. In Algorithm 2, $\alpha=2$ and $\kappa=\left(\sigma_{r}\right)^{2}$. Following the ordering of $\kappa$, the sequence $\sigma_{t}^{\prime}=t_{2} t_{1} t_{1} t_{2} t_{3}$ is fired, leading to the initial marking. Finally, after the initial firing of $t_{3}$, the sequence $\sigma_{t} \sigma_{t}^{\prime}=t_{4} t_{1} t_{4} t_{1} t_{2} t_{1} t_{1} t_{2} t_{3}$ returns to the initial marking.

The next corollary provides the characterization of reversibility for all live Equal-Conflict systems and is illustrated in Figure 10.

Corollary 1. Consider a live Equal-Conflict system $S=\left(N, M_{0}\right)$ such that $N=(P, T, W)$. The system $S$ is reversible if and only if it enables a T-sequence.

Proof. For the necessity, Lemma 1 applies. 
We prove the sufficiency next. Suppose there exists a feasible T-sequence $\sigma_{r}$ in the live system $S$. We show that after the firing of any feasible sequence $\sigma$, with $M_{0} \stackrel{\sigma}{\longrightarrow} M^{\prime}$, there exists a feasible sequence $\sigma^{\star}$ that leads to the initial marking. For that purpose, we show by induction on the length $n$ of $\sigma$ the property $P(n)$ :

"If a sequence $\sigma$ of length $n$ is feasible in a live Equal-Conflict system $S=\left(N, M_{0}\right)$ and a feasible T-sequence, denoted by $\sigma_{r}$, exists in $S$, then there exists a feasible sequence $\sigma^{\star}$ such that $M_{0} \stackrel{\sigma \sigma^{\star}}{\longrightarrow} M_{0} . "$

If $n=0, \sigma$ and $\sigma^{\star}$ are empty sequences and the initial marking is reached.

Otherwise, suppose $n>0$, with $\sigma=t \sigma^{\prime}$, note $M_{0} \stackrel{t}{\longrightarrow} M \stackrel{\sigma^{\prime}}{\longrightarrow} M^{\prime}$, and assume that the property $P(n-1)$ is true. Applying Theorem 2, there exists a sequence $\sigma_{t}^{\prime}$ that is feasible at $M$ such that $M \stackrel{\sigma_{t}^{\prime}}{\longrightarrow} M_{0}$ and the sequence $t \sigma_{t}^{\prime}$ is a T-sequence. Thus, the T-sequence $\sigma_{t}^{\prime} t$ is feasible at $M$. Applying the induction hypothesis on the sequence $\sigma^{\prime}$ of size $n-1$, which is feasible in the live system $(N, M)$, we obtain a sequence $\sigma_{d}$ that is feasible at $M^{\prime}$ and returns to $M$. Thus, the sequence $\sigma^{\star}=\sigma_{d} \sigma_{t}^{\prime}$ is feasible at $M^{\prime}$ and leads to $M_{0}$.

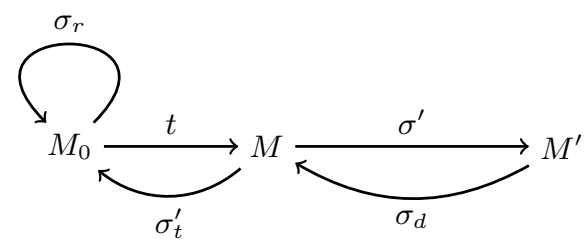

Fig. 10. If the T-sequence $\sigma_{r}$ and the sequence $\sigma=t \sigma^{\prime}$ are feasible at $M_{0}$, then the sequence $\sigma^{\star}=\sigma_{d} \sigma_{t}^{\prime}$ is feasible at $M^{\prime}$ and leads to $M_{0}$.

We deduce that after the firing of any feasible sequence in $S$, there exists a feasible sequence that returns to the initial marking. We conclude that $S$ is reversible.

Some examples of the previous sections provide some insight into the conditions of this characterization. Indeed, Figure 4 pictures non-reversible systems that are Equal-Conflict, live and do not enable any T-sequence. Figure 5 depicts a non-reversible system that is Equal-Conflict, non-live and enables a T-sequence.

\section{T-sequences in Larger Classes}

In the previous section, we showed that the existence of a feasible T-sequence is necessary and sufficient for reversibility in live Equal-Conflict systems, which are not necessarily bounded.

Now we provide some counter-examples for this condition in other subclasses of Petri nets. They are all strongly connected, live, and not reversible systems allowing a T-sequence. 
First, the characterization does not carry over to systems that are just wellformed, even if the net is ordinary, as shown in Figure 11, which is inspired from a system of [5].
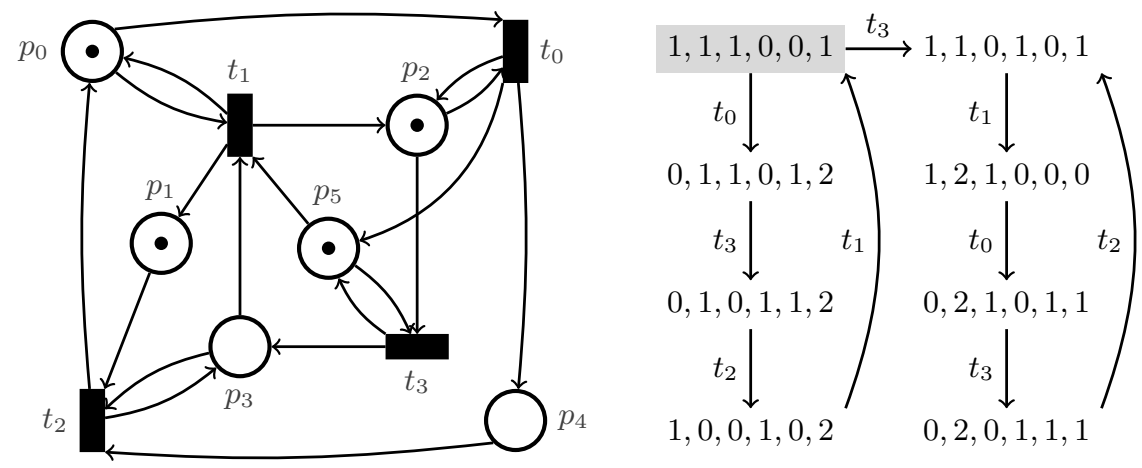

Fig. 11. The system allows the T-sequence $t_{0} t_{3} t_{2} t_{1}$. Liveness, boundedness and nonreversibility of the system can be deduced from its reachability graph on the right. Multiplying the input and output weights of $p_{0}$ by 2 yields a system in which any transition firing preserves the overall number of tokens. We deduce that $(2,1,1,1,1,1)^{T}$ is a conservativeness vector, hence the net is structurally bounded. Since it is also structurally live, it is well-formed.

Second, it does not apply either to the class of ordinary Asymmetric-Choice systems, as shown by the unbounded system of Figure 12.

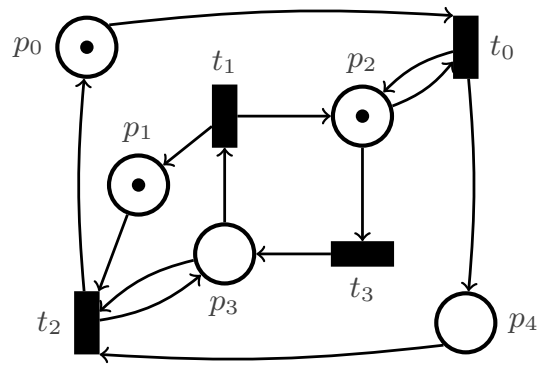

\begin{tabular}{c|c} 
Place $p$ & Output set $p^{\bullet}$ \\
\hline$p_{0}$ & $t_{0}$ \\
$p_{1}$ & $t_{2}$ \\
$p_{2}$ & $t_{0}, t_{3}$ \\
$p_{3}$ & $t_{1}, t_{2}$ \\
$p_{4}$ & $t_{2}$
\end{tabular}

Fig. 12. This ordinary Asymmetric-Choice system is unbounded since the place $p_{1}$ is unbounded (fire $\left(t_{3} t_{1}\right)^{\alpha}$ for any positive integer $\alpha$ ). It is live since $t_{1}$ and $t_{3}$ can always be fired after a finite number of firings, thus allowing new firings of $t_{0}$ and $t_{2}$. It is not reversible since there is always an occurrence of $t_{1}$ between two occurrences of $t_{2}$. The system allows the T-sequence $t_{0} t_{3} t_{2} t_{1}$.

Last, it does not extend to weighted Free-Choice systems, even when they are very close to Join-Free, as illustrated in Figure 13 where the system has only 
one synchronization - a join-transition with just two inputs - that distinguishes it from the Join-Free class. We have not found yet a counter-example belonging to the Join-Free class.

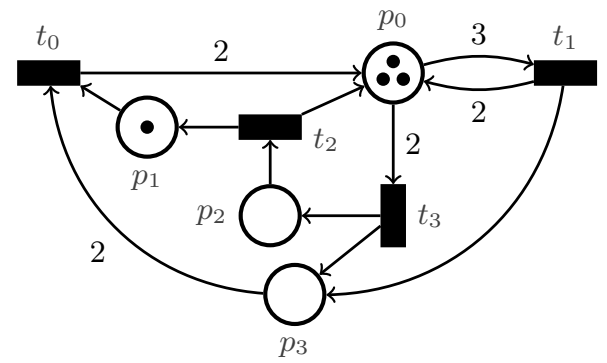

Fig. 13. In this weighted Free-Choice system, the T-sequence $t_{1} t_{3} t_{2} t_{0}$ is initially enabled. The place $p_{1}$ is unbounded (fire the sequence $\left(t_{3} t_{2} t_{3} t_{2} t_{0}\right)^{\alpha}$ for any positive integer $\alpha$ ), thus the system is unbounded. Two consecutive firings of $t_{1}$ are not possible, and $t_{0}$ is either enabled by a firing of $t_{1}$ followed by a firing of $t_{3}$, or by two firings of $t_{3}$ with a firing of $t_{2}$ in between. Firing only occurrences of $t_{2}$ and $t_{3}$ generates tokens in $p_{1}$ that cannot be destroyed. Hence the system is not reversible. After any firing sequence, it is possible to send three tokens back to $p_{0}$ while $p_{1}$ contains one or more tokens. Such a marking enables the T-sequence and we deduce the liveness.

\section{Conclusion}

In any weighted Petri net, the existence of a feasible T-sequence is necessary to have both liveness and reversibility, which are fundamental behavioral properties for embedded and flexible manufacturing systems and other real-world applications. This necessary condition was already known to be sufficient for well-formed, strongly connected, weighted Choice-Free and ordinary Free-Choice systems. It is no longer sufficient for the well-formed homogeneous S-systems, a class with both choices and weights included in the Equal-Conflict class.

By taking the liveness property as an assumption, we relaxed this condition and proved that it is sufficient for reversibility in all the live Equal-Conflict systems. Petri nets of this expressive class may not be strongly connected nor be bounded.

Finally, we exhibited several counter-examples, all live, non-reversible and allowing a T-sequence, belonging to larger classes of Petri nets.

As a consequence, extensions of our new characterization of reversibility would require more constraints. We believe that non-homogeneous Join-Free nets, bounded or not, or homogeneous bounded Asymmetric-Choice nets are worth investigating. 


\section{References}

1. Araki, T., Kasami, T.: Decidable Problems on the Strong Connectivity of Petri Net Reachability Sets. Theoretical Computer Science 4(1), 99-119 (1977)

2. Barkaoui, K., Couvreur, J.M., Klai, K.: On the Equivalence Between Liveness and Deadlock-Freeness in Petri Nets. In: Ciardo, G., Darondeau, P. (eds.) Applications and Theory of Petri Nets 2005, LNCS, vol. 3536, pp. 90-107. Springer Berlin Heidelberg (2005)

3. Best, E., Darondeau, P.: Petri Net Distributability. In: Clarke, E., Virbitskaite, I., Voronkov, A. (eds.) Perspectives of Systems Informatics, LNCS, vol. 7162, pp. 1-18. Springer, Heidelberg (2012)

4. de Frutos Escrig, D., Johnen, C.: Decidability of Home Space Property. Tech. Rep. LRI-503, Univ. de Paris-Sud, Centre d'Orsay, LRI (1989)

5. Desel, J., Esparza, J.: Free Choice Petri Nets, Cambridge Tracts in Theoretical Computer Science, vol. 40. Cambridge University Press, New York, NY, USA (1995)

6. Haddad, S., Mairesse, J., Nguyen, H.T.: Synthesis and Analysis of Product-form Petri Nets. Fundamenta Informaticae 122(1-2), 147-172 (2013)

7. Hujsa, T., Delosme, J.M., Munier-Kordon, A.: On the Reversibility of WellBehaved Weighted Choice-Free Systems. In: Ciardo, G., Kindler, E. (eds.) Application and Theory of Petri Nets and Concurrency, LNCS, vol. 8489, pp. 334-353. Springer International Publishing (2014)

8. Lee, E.A., Messerschmitt, D.G.: Synchronous Data Flow. Proceedings of the IEEE $75(9), 1235-1245$ (1987)

9. Lopez-Grao, J.P., Colom, J.M.: Structural Methods for the Control of Discrete Event Dynamic Systems - The case of the Resource Allocation Problem. In: Seatzu, C., Silva, M., van Schuppen, J.H. (eds.) Control of Discrete-Event Systems, Lecture Notes in Control and Information Sciences, vol. 433, pp. 257-278. Springer London (2013)

10. Memmi, G., Roucairol, G.: Linear Algebra in Net Theory. In: Brauer, W. (ed.) Net Theory and Applications, LNCS, vol. 84, pp. 213-223. Springer, Heidelberg (1980)

11. Murata, T.: Petri Nets : Properties, Analysis and Applications. Proceedings of the IEEE 77(4), 541-580 (1989)

12. Recalde, L., Teruel, E., Silva, M.: $\{\mathrm{SC}\}{ }^{*} \mathrm{ECS}$ : A Class of Modular and Hierarchical Cooperating Systems. In: Billington, J., Reisig, W. (eds.) Application and Theory of Petri Nets 1996, LNCS, vol. 1091, pp. 440-459. Springer Berlin Heidelberg (1996)

13. Sifakis, J.: Structural Properties of Petri Nets. In: Winkowski, J. (ed.) Mathematical Foundations of Computer Science, LNCS, vol. 64, pp. 474-483. Springer, Heidelberg (1978)

14. Teruel, E., Chrzastowski-Wachtel, P., Colom, J.M., Silva, M.: On Weighted Tsystems. In: Jensen, K. (ed.) Application and Theory of Petri Nets 1992, LNCS, vol. 616, pp. 348-367. Springer, Heidelberg (1992)

15. Teruel, E., Colom, J.M., Silva, M.: Choice-Free Petri Nets: A Model for Deterministic Concurrent Systems with Bulk Services and Arrivals. IEEE Transactions on Systems, Man and Cybernetics, Part A 27(1), 73-83 (1997)

16. Teruel, E., Silva, M.: Liveness and Home States in Equal-Conflict Systems. In: Marsan, M.A. (ed.) Application and Theory of Petri Nets 1993, LNCS, vol. 691, pp. 415-432. Springer, Heidelberg (1993)

17. Teruel, E., Silva, M.: Structure Theory of Equal Conflict Systems. Theoretical Computer Science 153(1\&2), 271-300 (1996) 